\title{
LIPID METABOLISM IN ACTIVE CROHN'S DISEASE: PRE-RESULTS
}

\author{
Vladimir Hrabovsky , Zdenek Zadak ${ }^{\mathrm{b}}$, Vladimir Blahac, Radomir Hyspler ${ }^{\mathrm{c}}$, Alena Ticha ${ }^{\mathrm{c}}$, \\ Tomas Karlik $^{\mathrm{d}}$
}

\author{
a Intensive Care Unit, Department of Internal Medicine, Faculty Hospital Ostrava, Czech Republic \\ ${ }^{b}$ Department of Research and Development, Charles University Prague, Faculty of Medicine Hradec Králové, Czech \\ Republic \\ c Department of Metabolic Care and Gerontology, Faculty Hospital Hradec Králove, Czech Republic \\ d Department of Clinical Biochemistry, Faculty Hospital Ostrava, Czech Republic \\ e-mail: vladimir.hrabovsky@fnspo.cz
}

Received: October 18, 2006; Accepted: November 11, 2006

Keywords: Inflammation/Lipids/Nutrition

Background: Crohn's disease (CD) is a chronic relapsing disease. Especially acute period may be associated with metabolic disturbances. Alteration of lipid metabolism has been described in critically ill patients and hypocholesterolemia is associated with poor prognosis. The human organism acquires cholesterol by two principal processes - synthesis de novo, and absorption from the diet. It is possible to assess, using cholesterol synthesis markers (lathosterol) and cholesterol absorption markers (sitosterol, campesterol) the leading form of cholesterol acquisition.

Aim: The aim of this study is assess the association between the lipid profile in plasma and the plasma concentration of sterols in active CD patients and in control subjects.

Method: Routine laboratory tests, CDAI, lipid and non-cholesterol sterols plasma levels were performed on days 3, 14 and 28. The metabolic parameters have been compared with a control cohort of 100 healthy blood donors.

Results: Presently, complete data for 8 patients are available The serum total cholesterol, LDL and HDL cholesterol, and triglyceride concentrations were lower in patients with acute Crohn's disease than in the control group. Moreover lathosterol, campesterol and sitosterol concentrations were lower, whereas squalene concentration was higher than in controls. As mentioned above, complete data are not currently available. Therefore statistical analysis has not been finished.

Conclusion: Our pre-results show substantial abnormalities in the concentrations of plasma lipids and non-cholesterol sterols, which are presented as markers of cholesterol requirement, in patients with acute CD.

\section{INTRODUCTION}

Crohn's disease (CD) is a chronic inflammatory process that may affect any part of the gastrointestinal tract. The etiology of the disease is still unknown and therefore treatment of the cause is problematic. Moreover, symptomatic treatment can be accompanied by serious complications. Patients will often present with various symptoms (e.g. abdominal pain, fever, and diarrhoea with or without bleeding). Up to $70 \%$ of patients with active Crohn's disease suffer from malnutrition and metabolic disturbances. Depletion in energy stores, catabolism, hypocholesterolemia, hypoproteinemia (alteration of signal and transport mechanisms), and water and electrolyte disturbances may play important roles in the course of the disease. Hypocholesterolemia is a common feature in patients with various types of acute disease. Alteration in lipid metabolism has been described in patients after trauma, surgery, burn injury or in sepsis and low level of cholesterol in critically ill patients is associated with poor prognosis ${ }^{1-3}$. Hypocholesterolemia in the acute state is connected with adrenal insufficiency ${ }^{4}$. Hypocholesterolemia, however, is a more complex phenomenon, quantifiably related to severity of illness ${ }^{5}$. The main cause of hypocholesterolemia in acute illnesses is inability to increase cholesterol synthesis according to requirement. Additional causes are the effect of the inflammation response, cytokines, hemodilution and a low intake of cholesterol during enteral and parenteral nutritional support ${ }^{6-8}$.

Although the relationship between hypocholesterolemia and acute illness is known, most studies on lipid and fatty acid profile in patients with $\mathrm{CD}$ have not reported changes in lipid metabolism related to changes in the cholesterol absorption/synthesis process. The human organism obtains cholesterol by two principal processes: synthesis de novo, and absorption from the diet. It is known that cholesterol synthesis markers (lathosterol and the lathosterol /cholesterol ratio in blood plasma), and cholesterol absorption markers (sitosterol and campesterol in blood plasma) can indicate both the dominant process in cholesterol acquisition and its degree ${ }^{9-11}$. The aim of this study was therefore to assess the association between the lipid profile in plasma and the plasma concentration of sterols as markers of the cholesterol synthesis/absorption 
process during the intensive care period in patients with active Crohn's disease and in control subjects.

\section{MATERIAL AND METHODS}

The Ethics Committee of the Faculty Hospital in Ostrava approved the study protocol, and all subjects gave their informed consent before the start of the study.

The study included 21 patients with active $C D$, whose Crohn's Disease Activity Index was more than 150 (CDAI $>150$ ). Patients were admitted to the Faculty Hospital in Ostrava and treated according to a standard protocol, based on usage of high doses of corticosteroids, mesalazine, vitamin E, antibiotics and symptomatic therapy. Enteral oligomeric nutritional support (no "home made diet") was used, and where necessary, parenteral nutrition support without the fat components in the nutrition protocol. The basic characteristics of these patients are shown in Table 1.

The metabolic parameters of each patient were evaluated by comparison with a control cohort of 100 healthy blood donors. This cohort reflected the polymorphism of cholesterol metabolism in the population. Table 5.

Routine laboratory tests and disease activity using Crohn's disease activity index (CDAI) were performed after admission to hospital. Plasma concentrations of total cholesterol, triglycerides, HDL and LDL cholesterol were measured enzymatically (enzymatic colour test with reagents Olympus OSR6216, 6133,6287,6183, Olympus System Calibrator) and analysed by automated chemistry analyzer (Olympus AU 2700, Japan)

Non-cholesterol sterols were determined in EDTAblood plasma samples, stored at $-80{ }^{\circ} \mathrm{C}$ until analysis. Sterols were extracted by Abell-Kendall procedure (alkaline hydrolysis by ethanolic solution of $\mathrm{KOH}$, followed by triple extraction with hexane), derivatised to trimethylsilylethers using BSTFA (Supelco, Bellefonte, USA) and analysed by gas chromatograpy - mass spectrometry (TurboMass, Perkin-Elmer, Wellesley, USA). All laboratory tests were performed on days 3, 14 and 28 .

\section{PRE-RESULTS}

To date, complete data for 8 patients are available. Others are under continuous investigation. Basic metabolic characteristics of these patients are shown in Table 2.

The serum total cholesterol, LDL and HDL cholesterol, and triglyceride concentrations were lower in patients with acute Crohn's disease than in the control group. In patients with active Crohn's disease, lathosterol, campesterol and sitosterol concentrations were lower, whereas squalene concentration was higher than in controls. Results are presented in Tables 3 and 4.

As mentioned above, complete data are not currently available. Therefore statistical analysis has not been done.
Table 1. Characteristics of patients at the start of study. CDAI-Crohn Disease Activity Index

\begin{tabular}{|l|c|}
\hline Patients & 8 \\
\hline Male & 5 \\
\hline Female & 3 \\
\hline Terminal ileitis & 2 \\
\hline Colitis & 6 \\
\hline Age & 24 \\
\hline Weight loss $(\mathrm{kg})$ & 6.4 \\
\hline CDAI & 277 \\
\hline
\end{tabular}

Table 2. Metabolic characteristics of patients at the start of study.

HCT - haematocrit, TLC - total lymphocyte count ALB - albumine, PreA - prealbumine, Fe - iron, $\mathrm{Ca}$ - calcium

\begin{tabular}{|c|c|c|c|c|c|c|}
\hline HCT & TLC & $\begin{array}{c}\text { Alb } \\
\mathrm{g} / 1\end{array}$ & $\begin{array}{c}\text { PreA } \\
\mathrm{g} / 1\end{array}$ & $\begin{array}{c}\mathrm{CRP} \\
\mathrm{mg} / 1\end{array}$ & $\begin{array}{c}\mathrm{Fe} \\
\text { umol/1 }\end{array}$ & $\begin{array}{c}\mathrm{Ca} \\
\mathrm{mmol} / 1\end{array}$ \\
\hline 0.34 & 0.98 & 29.7 & 0.18 & 58 & 6.2 & 2.14 \\
\hline
\end{tabular}

Table 3. Plasma concentration of lipids (mmol/1) t-CH total cholesterol, TGC triglycerides.

\begin{tabular}{|l|c|c|c|c|}
\hline & day 0 & day 3 & day 14 & day 28 \\
\hline t-CH & 3.8 & 2.88 & 4.37 & 4.87 \\
\hline LDL-CH & 2.04 & 1.29 & 2.05 & 2.49 \\
\hline HDL-CH & 1.02 & 1.01 & 1.31 & 1.58 \\
\hline TGC & 2.17 & 1.3 & 1.91 & 1.72 \\
\hline
\end{tabular}

Table 4. Plasma concentration of sterols (umol/1) SQ squalene, LTH lathosterol, SIT sitosterol CAM campesterol.

\begin{tabular}{|c|c|c|c|}
\hline & day 3 & day 14 & day 28 \\
\hline SQ & 1.7 & 1.5 & 1.35 \\
\hline LTH & 1.3 & 0.97 & 1.01 \\
\hline SIT & 4.6 & 6.3 & 5.98 \\
\hline CAM & 2.07 & 3.53 & 3.7 \\
\hline
\end{tabular}

Table 5. Characteristics of controls SQ squalene, LTH lathosterol, SIT sitosterol CAM campesterol t-CH total choleterol.

\begin{tabular}{|c|c|c|c|c|}
\hline \multicolumn{5}{|c|}{ control group } \\
\hline SQ & LTH & SIT & CAM & t-CH \\
\hline umol/1 & umol/1 & umol/1 & umol/1 & $\mathrm{mmol} / 1$ \\
\hline 1.17 & 7.8 & 5.03 & 10.22 & 4.91 \\
\hline
\end{tabular}




\section{DISCUSSION}

Active Crohn's disease is frequently associated with malnutrition. Our results show weight loss of an average $6,4 \mathrm{~kg}$ during 3 months. The main cause of malnutrition in patients with $\mathrm{CD}$ is anorexia, which seems to be a result of the production of inflammatory cytokines. Elevation of proinflammatory cytokines such as interferon- $\gamma$, interleukin (IL) -1,IL-6,IL-12 and tumor necrosis factor-alpha ( TNF- $\alpha$ ) has been demonstrated in patients with Crohn's disease ${ }^{12}$. Digestion and absorption impairment, protein-losing enteropathy, and high energy expenditure are further factors which can be included in the etiology of malnutrition in patients with CD. Moreover, all these must be taken into account during the process of determination and formation of a nutrition strategy. Suppression of catabolism by adequate energy supply, substitution for depletion of macronutrients and micronutrients, normalisation of water/electrolyte disturbances and potential use of immunonutrition are the grand components of treatment in Crohn's disease. Although corticosteroids are more effective than enteral nutrition in inducing and sustaining remissions in patients with acute $\mathrm{CD}$, enteral nutrition has been established as an inseparable part of primary treatment of active $\mathrm{CD} \cdot{ }^{13,14}$.

Recently, the fat composition of enteral nutrition has been the subject of interest. Fatty acids have been recognized as components, which are able to modulate gut-associated lymphoid tissue (GALT) functions ${ }^{15,16}$. Therefore, major importance has been given to determining the relationship between nutritional fat intake, intestinal mucosal immunity and severity of disease. Results of recent studies show that a high content of long-chain fatty acids in nutrition support may have negative influences on the overall positive effect of enteral nutrition ${ }^{17}$. There are also known immunomodulatory and anti-inflammatory effects of $\omega$-3 poly-unsaturated fatty acids (PUFA) in CD (18).

Alteration in the lipid, fatty acid and lipoprotein profile has been detected in patients with active CD before. Further, diminished antioxidant status was also observed ${ }^{19,20}$. The cause of hypocholesterolemia has been mentioned above. Depletion of poly-unsaturated fatty acids is the result of high consumption of these substrates for reparation of damaged tissues and conversion into eicosanoids. Our pre-results have also identified lower serum levels of total cholesterol and LDL and HDL cholesterol in comparison with the control group. Although published data appear to indicate higher triglyceride concentrations in the blood of CD patients, triglyceride concentration was lower in our study. Triglycerides are the most important lipids in food, and they are considered a main source of energy in humans, not only during disease. However, the artificial nutrition fat content can affect serum triglyceride levels.

Although a relationship between hypocholesterolemia and acute illness has been established, most studies on lipid and fatty acid profile in patients with acute $C D$ have not reported changes in lipid metabolism in connection with changes in the cholesterol absorption/synthesis process. The human organism acquires cholesterol by two principal processes - synthesis de novo, and absorption from the diet. It is possible to assess, using cholesterol synthesis markers (lathosterol in blood plasma) and cholesterol absorption markers (sitosterol, campesterol in blood plasma) the leading form of cholesterol acquisition ${ }^{21}$. We observed lower levels of lathosterol and low values of lathosterol/cholesterol ratio. This is an important presumption for the alteration of cholesterol synthesis in acute CD. The observed lower concentrations of campesterol and sitosterol at the start of study in patients with acute CD could be explained by a reduction of "home made diet" intake. Later, elevation of these sterols on day 14 and 28 is caused by restitution of dietary intake. Squalene plasma level was higher as a result of release of squalene from fat stores during acute starvation.

\section{CONCLUSION}

The aim of the project is to find the dependency of serious hypolipidemia (especially hypocholesterolemia) in patients with active Crohn's disease on the activity of disease and the source of cholesterol metabolism (synthesis $\mathrm{x}$ absorption). Our pre-results show substantial abnormalities in the concentrations of plasma lipids and non-cholesterol sterols, which are presented as markers of cholesterol requirement. However, complete data are not available and the final statistical evaluation has not been done. Thus, more extensive research is required to describe the metabolism of lipids and especially cholesterol, as a substance necessary for cell membrane reparation and synthesis of steroid hormones, and whose importance for survival of stress is well known. If the phenomenon of hypocholesterolaemia would be elucidated and quantitative data about cholesterol synthesis would be obtained, the possibility of parenteral nutrition supplementation by cholesterol or its precursors should be realized.

\section{ACKNOWLEDGEMENT}

Supported by grant IGA Ministry of Health Czech Republic No NR 7854-3.

\section{REFERENCES}

1. Giovannini I, Boldrini G, Chiarla C, Guiliante F, Vellone M, Nuzzo G. Pathophysiologic correlates of hypocholesterolemia in critically ill surgical patients. Int Care Med 1999; 25:748-51

2. Bakalář B, Zadák Z, Pachl J,Hyšpler R,Crhová S. Influence of severe trauma on cholesterol synthesis. Int Care Med 2000; 26, S3:357

3. Fraunberger P, Nagel D, Walli AK,Seidel D. Serum cholesterol and mortality in patients with multiple organ failure. Crit Care Med 2000; 28:3574-5

4. Bakalář B, Zadák Z, Pachl J, Hyšpler R, Crhová Š, Karásek J, Capek V. Severe hypocholesterolemia is associated with adrenal insufficiency in multiple trauma patients. Int Care Med 2001; 27, Suppl 2:253.

5. Giovannini I, Chiarla C, Greco F, Boldrini G, Nuzzo G. Characterization of biochemical and clinical correlates of hy- 
pocholesterolemia after hepatectomy. Clin Chemistry 2003; 49: 317-320

6. Gordon BR, Parker TS, Levine DM, Saal SD, Wang JCL, Sloan B-J,at al. Relationship of hypolipidemia to cytokine concentrations and outcomes in critically ill patients. Crit Care Med 2001; 29:1563-1568

7. Bonville DA, Parker TS, Levine DM, Gordon BR, Hydo LJ, Eachempati SR, et al. The relationship of hypocholesterolemia to cytokine concentrations and mortality in critically ill patients with systemic inflammatory response syndrome. Surg Infect 2004; 5:39-49

8. Zadák Z, Hyšpler R, Havel E, Bláha V, Sobotka L, Žd’ánský P, et al. Changes in metabolism of cholesterol and its precursors in critical patients on total parenteral nutrition. Nutrition 2002; 18:214

9. Tilvis RS, Miettinen TA. Serum plant sterols and their relation to cholesterol absorption. Am J Clin Nutrition

10. Pfohl M, Naoumova RP, Kim KD et al. Use of cholesterol precursors to assess changes in cholesterol synthesis under non.steadystate conditions. Eur J Clin Ivestig 1998; 28:491-496.

11. Barton BE. IL-6-like cytokines and cancer cachexia: consequences of chronic inflammation. Immunology research 2001; 23:41-58.

12. McLean SJ, Rombeau JL. Cytokines and inflammatory bowel disease: a review. Journal of parenteral and enteral nutrition 1998 23:20-24.

13. Vointk AJ, Echave V, Feller JH, Brown RA, Gurd FN. Experience with elemental diet in the treatment of inflammatory bowel disease. Is this primary therapy? Arc Surg 1973; 107:329-333.
14. Greenberg GR, Fleming CR, Jeejeebhoy KN, et al.Controlled trial of bowel rest and nutrition support in the management of Crohn's disease. Gut 1998; 29:1309-1315.

15. Gassul MA, Fernandez-Baňares F, Cabre E. et al. Fat composition may be a clue to explain the primary therapeutic effect of enteral nutrition in Crohn's disease: results of a double blind randomized multicentre European trial. Gut 2002; 51:164-168.

16. Miura S, Tsuzuki Y, Hokari R, Ishii H. Modulation of intestinal immune system by dietary fat intake: Relevance to Crohn disease. J Gastroenterol Hepatol 1998; 13:1183-1190.

17. Bamba T, Shimoyama T,Sasaki M, Tsujikawa T, Fukuda Y, Koganei $\mathrm{K}$, at al. Dietary fat attenuates the benefit of an elemental diet in active Crohn's disease: a randomized, controlled trial. Eur J Gastroenterol Hepatol 2003; 15:151-157.

18. Belluzi A, Brignota C, Campieri M. et al.: Effect of an entericcoated fish-oil preparation on relapses in Crohn's disease. N Eng J Med 1996; 334:1557-1560.

19. Levy E, Rizwan Y, Thibault L, Lepage G, Brunet S., Bouthillier L, et al. Altered lipid profile, lipoprotein composition, and oxidant and antioxidant status in pediatric Crohn disease. Am J Clin Nutr 2000; 71:807-815.

20. Geerling BJ, v. Houwelingen AC, Badart-Smook A, Stockbrügger RW, Brummer RJM. The relation between antioxidant status and alterations in fatty acid profile in patients with Crohn disease and controls. Scand J Gastroenterol 1999; 11:1108-1116.

21. Kuksis A. Plasma non-cholesterol sterols. J Chromatogr 2001; 935:203-236. 